\title{
A demonstration of shape constancy
}

\author{
JOSEPH S. LAPPIN and LAURENCE D. PREBLE \\ Vanderbilt University, Nashville, Tennessee 37240
}

\begin{abstract}
In order to compare the recognition of objective (defined in three dimensions) and projective (on the frontal-parallel plane) shapes, subjects were asked to identify angles (either objective or projective) on random polygons that were displayed in complex and meaningful photographic slides in the frontal-parallel plane. The subjects' judgments corresponded much more closely to the objective than to the projective shapes, almost independently of which of the two shapes they were asked to judge and of whether the slides were presented in a normal upright orientation or were rotated 180 deg. The results are incompatible with the strong form of the shape-slant hypothesis, which assumes that the primary perceptual information concerns the properties of the projective retinal image. The results indicate, instead, that a tacit conception of three-dimensional space is primary in the perceptual process.
\end{abstract}

Shape constancy is an important phenomenon in the study of visual perception. It refers to the fact that perceptual knowledge is obtained about the three-dimensional shapes of environmental stimulus objects, approximately invariant over the transformation group of two-dimensional shapes projected on the retina. Shape constancy represents one of the principal illustrations of perception as an invariance over specific sensory data; as such, it is familiar to students in introductory psychology as well as to researchers in visual perception.

Familiarity often breeds neglect, however. Advanced textbooks on perception typically contain no more discussion of the phenomenon than can be found in most introductory texts, and reference works often avoid the problem entirely. Experimental analyses of shape constancy must be regarded as having contributed very little to our understanding of the phenomenon. Good reviews of the available literature have been provided by Epstein and Park (1963). Hake (1966), and Hochberg (1972). Epstein and Park conclude their review with the comment that "...there is surprisingly little in the way of well established functional relationships in this area. With the possible exception of size constancy theoretical speculation has far outdistanced (or disregarded) the experimental evidence" (p. 287). Similarly, Hake (Note 1) has suggested that "... our present understanding of perceptual constancy is not much more than legendary." Traditional beliefs about the invariance of form perception have been more influential than the findings of research.

Laboratory investigations of shape constancy have usually produced evidence about the failure of ohservers to achieve constancy. The predominant

This study was supported in part by Public Health Service Grant MH-21105. The study was conducted by the second author as part of an Honors thesis in Psychology. The authors are grateful to Brian Kottas for his help in several phases of the experiment. finding has been that the perceived shape tends to be intermediate between the objective and projective shapes; Thouless (1931) referred to this perceptual result as a "compromise" between the two extremes. The degree to which this "compromise" approaches the objective shape has been found to depend upon the availability of cues to depth, the degree of slant from the frontal-parallel plane, the observer's attitude (objective, retinal, or apparent), and several other variables (see Epstein \& Park, 1963). These demonstrations of the limitations of perception have, however, failed to determine the critical conditions under which shape constancy can be reliably achieved-largely because shape constancy has rarely been produced in the laboratory. The numerous demonstrations of significant deviations from perceptual constancy have even led Hake (1966) to suggest that "this invariance of form in vision has been exaggerated in the thinking of lay persons and professional persons interested in the problem" (p. 151). If indeed the phenomenon vanishes under controlled laboratory conditions, then experimental analyses are likely to be unproductive. Perhaps, however, the laboratory conditions have been too deprived: Simple stimuli such as circles, ellipses, rectangles, trapezoids, etc., have typically been viewed in plain, uncluttered backgrounds from which most potential depth cues have been removed. Perhaps it would be more fruitful to study the perception of shapes in more complex but ecologically more valid stimulus environments.

The experimental paradigm for investigating shape constancy has been constructed primarily around the "shape-slant invariance hypothesis." Sometimes this has been interpreted simply as a general hypothesis that the retinal image simultaneously determines both a perceived shape and its perceived slant (e.g., Beck \& Gibson, 1955); but the more common interpretation seems to have been a stronger Helmholtzian hypothesis - that a three-dimensional shape is 
inferred from the two-dimensional shape on the retina plus its perceived slant away from the frontal-parallel plane as judged by available depth cues (see Epstein \& Parks, 1963, and Hochberg, 1972, for reviews of the rationale and evidence for the shape-slant invariance hypothesis). If the shape-slant invariance hypothesis is taken as an explanation rather than just a description of shape constancy, then an implicit corollary is that the subject's sensory information about his retinal image is more accessible and more reliable than his knowled ge about three-dimensional space. Most experimental tests of the hypothesis have focused on the dependence of shape judgments on cues to depth or slant, and on the hypothesized correlation between judgments of shape and slant. Almost no direct tests have been made, however, of the implicit assumption that subjects can reliably judge the shapes of their retinal images.

The aim of the present experiment was to compare the subject's accuracy in identifying objective shapes in three dimensions and projective shapes in two dimensions.

Our methodological strategy differed in several ways from previous studies: First, we used stimulus displays that were richer in depth cues than has been the case in most other studies. Specifically, subjects identitied objective and projective angles formed by the sides of random polygons that were lying on a table with a number of common objects; these relatively complex and meaningful stimulus displays were presented in full-color photographic slides.

Second, the objective and projective shapes (associated with the same angle on each polygon) were independently varied, and the subjects were asked in separate conditions to identify both objective and projective shapes of each stimulus. Accordingly, it was possible to determine the correlation of the subject's judgments with both objective and projective shape, independently of which property the subject was asked to judge. If shape constancy is a real and valid perceptual phenomenon, then judgments of objective shape should correlate more highly with objective three-dimensional shape than with projective two-dimensional shape. If objective shape is inferred from the two-dimensional retinal projection, then judgments of projective shape should also be more accurate than judgments of objective shape. On the other hand, if knowledge of objective three-dimensional shape is obtained directly and is invariant over specitic retinal images, then judgments of objective shape should be more accurate than those of projective shape, and both judgments might even be expected to correlate more highly with the actual objective shape than with the projective shape.

Third, as an additional index of whether the knowledge about three-dimensional space is derived from retinal geometry or from acquired deductions we presented the stimulus displays in both a normal upright orientation and in an inverted or upside-down orientation. If the perception of space wore dependent upon the familiarity of the spatial relations, then the relative accuracy of the judgments of objective shape would probably be poorer in the inverted viewing conditions; similarly, judgments of projective shape might be expected to be more accurate with the inverted than with the upright orientation.

\section{METHOD}

The stimuli were irregular octagons that were randomly gencrated with the constraint that one angle (interior) on each tigure was fixed at $65,75,85,95,105$, or $115 \mathrm{deg}$. Six different octagons, ${ }^{1}$ one for each angle, were used during the experimental trials. and a different set of six octagons was used during the practice trials.

The polygons were cut from blue construction paper, placed in the center of a white posterboard, and surrounded with objects such as might be found in an office (e.g. . books. scissors, pencil); a wall covered with the same posterboard was also visible at the edge of the table. The specitic angle to be judged was designated by a bright orange spot placed opposite the vertex, outside the figure. A black-and-white reproduction of one of the slides appears in Figure 1 .

The stimuli were photographed by a camera placed in a fixed position at a viewing angle of approximately 30 deg above the plane of the polygon. Each polygon was placed in the center of the display and rotated about the vertex of the designated angle until its projective angle attained the desired size $(65,75,85,95,105$, or 115 deg). These projective angles were measured by means of a television camera placed in the same position as the $35-\mathrm{mm}$ camera with which the displays were photographed. Color slides were made with Kodak Ektachrome- $X$ film. After development of the slides, the angles were checked and rephotographed as necessary so that the actual projective angle was within \pm 1.5 deg of the desired value. Thirty-six slides were used for the experimental series-one for each combination of objective and projective angle. A separate set of six slicles. of six different octagons photographed from directly overhead so that the objective and projective angles were equal, was used for the practice trials.

The subject and experimenter were in separate rooms. Communication was by intercom. The stimuli were viewed on a rear-projection screen located in the wall between the subject and experimenter. The projection screen was located in a plane perpendicular to the subject's line of sight at a distance of about $90 \mathrm{~cm}$. Viewing was binocular. The entire display subtended

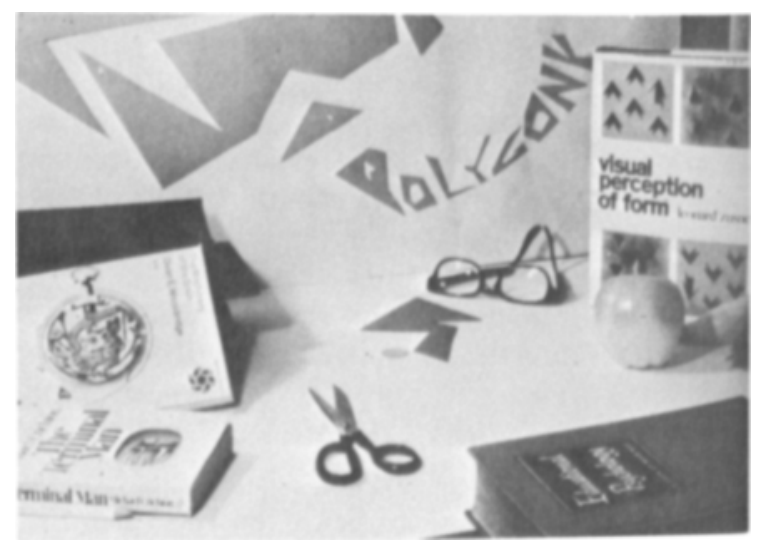

Figure 1. A black-and-white reproduction of one of the stimulus displays. In this case, the objective angle is $115 \mathrm{deg}$ and the projective angle is $65 \mathrm{deg}$. 
approximately 21 deg of visual angle in the horizontal direction and $15.5 \mathrm{deg}$ in the vertical direction. The test forms were about $3 \mathrm{deg}$ in diam.

The stimulus displays were presented for $1 / 2 \sec ^{2}$ and were initiated by the subject, with a $1 / 2$-sec delay between depression of the switch and onset of the slide. Control and timing of the stimulus presentations were accomplished by Iconix logic and timing equipment connected to a Lafayette tachistoscopic shutter on a (irousel projector.

\section{Subjects}

Fight undergraduate students at Vanderbilt University served as paicl roluntecrs.

\section{Procedure}

Fach subject served for two sessions. Each session consisted of wo blacks of 30 trials-one block devoted to judgments of objective angles and the other to judgments of projective angles. Each combination of the six objective and six projective angles was presented once during the block of 36 trials, with the order of presentation randomized for each block of trials. In one of the two sessions, the slides were rotated $180 \mathrm{deg}$ so that the scene was inverted tron its normal orientation. The ordering of the two- and three-dimensional judgment conditions and of the upright and inverted vicwing conditions was counterbalanced across subjects.

The task was thoroughly explained to the subject at the beginning of the tirst session. Care was taken that the subject should understand the distinction between the objective and projective angles formed by sides of the polygon. and the explanation was repeated and paraphrased until the experimenter was contident that the subject understood. Simple schematic drawings were also used to illustrate the basic idea about the projective geometry. The subject was told that the angles would be $65,75,85,95,105$, or $115 \mathrm{dcg}$ and that he should choose one of the six alternatives on each trial. At least 12 practice trials, in which each of the six alternatives occurred equally often, were used at the beginning of the first session to familiarize the subject with the task and with the angles to be judged. The six octagons used during the practice trials were photographed trom directly overhead in a homogeneous background with no depth cues, so that the objective and projective angles were equal. Since these polygons were different from those used during the experimental trials, the initial practice presumably provided equal training for both the objective and projective judgment conditions. Feedback specifying the correct response was given during the practice trials but not during the experimental trials.

\section{RESULTS}

The results are presented in Table 1, which gives the correspondence of each subject's judgments with the actual objective and projective shapes under each of the four conditions of judgment (objective vs. projective) or orientation (upright vs. inverted). The psychophysical correspondences between the judged and actual objective and projective angles were described in two ways-by the average absolute deviation (in degrees) between the judged and correct angles and by the percentage of variance in judgments accounted for by the actual objective and projective angles (i.e., by the values of $\mathrm{r}^{2}$ for the linear correlation between judged and actual angles).

As may be seen, the judgments of both objective and projective angles corresponded much more closely with the actual objective angles than with the projective angles for all subjects and all conditions. Of the 32 subject-by-condition combinations, there are only two in which the judgments corresponded more closely to the projective than to the objective angles, and in both cases the superiority was very slight and statistically insigniticant. In contrast, the objective correspondences were statistically superior in 19

Table 1

The Correspondences of Judged Angles With Actual Projective and Objective Angles

\begin{tabular}{|c|c|c|c|c|c|c|c|c|c|c|c|}
\hline & \multirow{2}{*}{$\begin{array}{l}\text { Judgment } \\
\text { Condition }\end{array}$} & \multirow{2}{*}{$\begin{array}{l}\text { Actual } \\
\text { Angle }\end{array}$} & \multicolumn{8}{|c|}{ Subjects } & \multirow[b]{2}{*}{ Average } \\
\hline & & & 1 & 2 & 3 & 4 & 5 & 6 & 7 & 8 & \\
\hline \multirow{12}{*}{$\begin{array}{l}\text { Absolute } \\
\text { Deviation } \\
\text { (Degrees) } \dagger\end{array}$} & \multirow{2}{*}{$\begin{array}{l}\text { Upright, } \\
\text { Objective }\end{array}$} & Projective & $\begin{array}{c}17.8 \\
* *\end{array}$ & $\begin{array}{c}22.5 \\
* *\end{array}$ & $\underset{* *}{16.1}$ & $\begin{array}{c}16.9 \\
* *\end{array}$ & $\begin{array}{c}15.0 \\
* *\end{array}$ & $\begin{array}{c}19.2 \\
* *\end{array}$ & 16.7 & $\underset{* *}{17.2}$ & 17.7 \\
\hline & & Objective & 9.4 & 8.6 & 5.6 & 10.3 & 12.8 & 6.4 & 11.7 & 6.1 & 8.9 \\
\hline & \multirow{2}{*}{$\begin{array}{l}\text { Upright, } \\
\text { Projective }\end{array}$} & Projective & $\begin{array}{c}19.4 \\
*\end{array}$ & 12.5 & 14.4 & $\underset{* *}{16.1}$ & 15.0 & $\begin{array}{c}18.3 \\
* *\end{array}$ & $\begin{array}{c}15.6 \\
* *\end{array}$ & 12.8 & 15.5 \\
\hline & & Objective & 12.2 & 13.6 & 8.9 & 5.6 & 8.3 & 5.0 & 7.2 & 10.0 & 8.8 \\
\hline & \multirow{2}{*}{$\begin{array}{l}\text { Inverted, } \\
\text { Objective }\end{array}$} & Projective & $\begin{array}{c}17.8 \\
* *\end{array}$ & $\begin{array}{c}19.4 \\
* *\end{array}$ & 13.6 & 12.5 & 15.8 & $\begin{array}{c}16.7 \\
* *\end{array}$ & $\begin{array}{c}15.8 \\
* *\end{array}$ & $\begin{array}{c}17.2 \\
* *\end{array}$ & 16.1 \\
\hline & & Objective & 55.6 & 5.0 & 10.3 & 8.6 & 9.7 & 7.8 & 6.4 & 7.8 & 7.6 \\
\hline & \multirow{2}{*}{$\begin{array}{l}\text { Inverted, } \\
\text { Projective }\end{array}$} & Projective & $\begin{array}{c}16.7 \\
* *\end{array}$ & 14.7 & 10.8 & $\begin{array}{c}13.9 \\
*\end{array}$ & 13.6 & $\begin{array}{c}16.7 \\
* *\end{array}$ & 13.1 & 15.6 & 14.4 \\
\hline & & Objective & 7.8 & 10.3 & 11.9 & 8.3 & 12.5 & 7.8 & 9.2 & 11.1 & 9.9 \\
\hline & $\begin{array}{l}\text { Upright, } \\
\text { Objective }\end{array}$ & $\begin{array}{l}\text { Projective } \\
\text { Objective }\end{array}$ & $\begin{array}{l}.00 \\
.40\end{array}$ & $\begin{array}{l}.03 \\
.62\end{array}$ & $\begin{array}{l}.03 \\
.72\end{array}$ & $\begin{array}{l}.01 \\
.28\end{array}$ & $\begin{array}{l}.08 \\
.23\end{array}$ & $\begin{array}{l}.01 \\
.77\end{array}$ & $\begin{array}{l}.00 \\
.36\end{array}$ & $\begin{array}{l}.00 \\
.72\end{array}$ & $\begin{array}{l}.02 \\
.52\end{array}$ \\
\hline & $\begin{array}{l}\text { Upright, } \\
\text { Projective }\end{array}$ & $\begin{array}{l}\text { Projective } \\
\text { Objective }\end{array}$ & $\begin{array}{l}.00 \\
.31\end{array}$ & $\begin{array}{l}.27 \\
.28\end{array}$ & $\begin{array}{l}.08 \\
.37\end{array}$ & $\begin{array}{l}.02 \\
.77\end{array}$ & $\begin{array}{l}.12 \\
.62\end{array}$ & $\begin{array}{l}.02 \\
.82\end{array}$ & $\begin{array}{l}.05 \\
.65\end{array}$ & $\begin{array}{l}.13 \\
.43\end{array}$ & $\begin{array}{l}.09 \\
.53\end{array}$ \\
\hline & $\begin{array}{l}\text { Inverted } \\
\text { Objective }\end{array}$ & $\begin{array}{l}\text { Projective } \\
\text { Objective }\end{array}$ & $\begin{array}{l}.00 \\
.79\end{array}$ & $\begin{array}{l}.00 \\
.77\end{array}$ & $\begin{array}{l}.15 \\
.34\end{array}$ & $\begin{array}{l}.13 \\
.54\end{array}$ & $\begin{array}{l}.02 \\
.33\end{array}$ & $\begin{array}{l}.00 \\
.63\end{array}$ & $\begin{array}{l}.02 \\
.64\end{array}$ & $\begin{array}{l}.00 \\
.65\end{array}$ & $\begin{array}{l}.04 \\
.59\end{array}$ \\
\hline & $\begin{array}{l}\text { Inverted, } \\
\text { Projective }\end{array}$ & $\begin{array}{l}\text { Projective } \\
\text { Objective }\end{array}$ & $\begin{array}{l}.03 \\
.58\end{array}$ & $\begin{array}{l}.09 \\
.48\end{array}$ & $\begin{array}{l}.31 \\
.22\end{array}$ & $\begin{array}{l}.17 \\
.58\end{array}$ & $\begin{array}{l}.18 \\
.35\end{array}$ & $\begin{array}{l}.03 \\
.62\end{array}$ & $\begin{array}{l}.21 \\
.41\end{array}$ & $\begin{array}{l}.06 \\
.35\end{array}$ & $\begin{array}{l}.14 \\
.45\end{array}$ \\
\hline
\end{tabular}


instances (at $\mathrm{p}<.05$, by the $\mathrm{t}$ test with $\mathrm{df}=35$, and also by the sign test for comparing the size of the deviations from the objective and projective angles.) Although the judgments seem to have been very little affected by whether the subjects were asked to identify two- or three-dimensional angles, there was a small but significant tendency for the judgments to be more closely related to the projective angles when the subjects were instructed to judge projective angles on the inverted slides than in any of the other conditions ( $p<.05$ by a binomial test on the average deviations, and $p<.01$ by the binomial test on the $r^{2}$ values). There were no significant main effects for either the objective vs. projective judgment conditions or for the upright vs. inverted viewing conditions.

\section{DISCUSSION}

The clear finding of this experiment was that shapes were perceived in a space that corresponded much more closely to the objectively valid three-dimensional space than to the frontal-parallel plane onto which the objective space was projected. In short, shape constancy was demonstrated.

Indeed, our subjects were profoundly inept at judging projective shapes. Even when explicitly asked to identify the projective shapes, their judgments were determined much more by the objective than by the projective shapes $-49 \%$ vs. $11 \%$ of the variance in projective judgments was determined, respectively, by the objective and projective shapes. This confusion of the objective for the projective shape is a rather compelling illusion. Two of our subjects (5 and 7) spontaneously complained that the $1 / 2$-sec exposure duration was too brief for them to identify the objective shape and that they could see only the projective shape in that time-in fact, however, the objective shapes accounted for $51 \%$ and the projective shapes $14 \%$ of the variance in their judgments of projective shape. This experiment also works quite effectively as a classroom demonstration.

The stimulus displays in this experiment were photographic projections onto a clearly defined plane surface perpendicular to the subjects' line of sight. Despite the obviousness of this planar projection, within a clear two-dimensional frame, the perceived shapes were evidently determined by reference to the three-dimensional space that was the subject of the photograph rather than by the plane of the photograph itself. If the subjects were so inaccurate at judging shapes by reference to this external frontal-parallel plane, then presumably they would do little or no better at judging shapes projected on their retinae. The difficulty in estimating the shapes of retinal images is easily confirmed simply by asking people to perform the task. (Although most college students do not readily understand what is being requested, they will usually catch on with the aid of schematic drawings.) Most people initially have great difficulty in deciding whether an angle that is objectively $90 \mathrm{deg}$ is projectively obtuse or acute.

The inability to accurately judge the shapes of retinal projections is incompatible with the version of the shape-slant invariance hypothesis that assumes that direct sensory knowledge of the shape of the retinal image is used as a basis for inferring the three-dimensional shape of environmental objects. The derivation of objective shape from the projective shape might, however, be based on preconscious perceptual processes rather than on conscious judgmental processes. Under this weaker version of the shape-slant hypothesis, the judgments of projective shape would not necessarily be expected to be more accurate than those of objective shape. This more general version of the hypothesis is probably acceptable to many more investigators than the stronger version that was tested in this experiment, but the weaker version loses most of its explanatory value. If the process for deriving objective shape from the retinal projection cannot be experimentally teased apart into distinguishable substages of information acquisition, then the shape-slant invariance hypothesis is essentially little more than a description of the phenomenon of shape constancy. Even this weaker version of the hypothesis is suspect, however, insofar as it specifies a linear combination of information from multiple sensory cues: Analysis of the projective geometry of the relation between the objective and projective angles in our task reveals that the relation is nonlinear and also depends on the orientation of the polygon in the slanted plane. In any case, it is appropriate to consider alternative hypotheses about how the objective shape might be derived from its projective image.

Shape constancy has usually been conceptualized as a phenomenon in which the perceptual system first operates on the projective image to resolve the cues to shape, depth, slant, etc., in order to then infer the structure of a three-dimensional space. The alternative to this approach would seem to be that the perceptual system functions to resolve among alternative three-dimensional rather than twodimensional spaces. That is, the visual system may utilize a tacit conception of three dimensions in which lines tend to be parallel rather than convergent, where angles on objects tend to be rectilinear rather than obtuse or acute, where textures and brightnesses are uniform rather than graded, etc. The perceptual task in interpreting a retinal image or a photograph would then be to determine the projective transformation that specifies the mapping of points between the three-dimensional space and the two-dimensional projection. The important point is that this projective transformation can be specified by only four 
parameters-three to determine the orientation of the plane within the three-dimensional space and a fourth to determine its distance from some reference point (sec Roberts, 1965). Thus, any given projective image specities a particular family of potential threedimensional spaces that could have produced this image. but ordinarily only a very restricted subset of these potential spaces would contain a permissible structure of parallel lines, right angles, uniform distributions of texture and brightness, etc. Complex real-world scenes, even as simple as those used in this experiment. contain many such relationships that can serve to specify the original three-dimensional space.

Perhaps the distinction of this hypothesis from the traditional shape-slant invariance hypothesis seems merely semantic when applied to the present problem of interpreting a single photographic representationsince the hypothesis of the primacy of three dimensions presumes that the perceptual system determines the slant of the projective plane in three-dimensional space and the shape-slant invariance hypothesis presumes that the perceptual sistem determines the slant of objective planes away from the frontal-parallel projective plane. The distinction between these hypotheses becomes more important. however, in dealing with the perception of an invariant space during motion of the observer's eres, head, and body. The geometric invariants under such motion are characteristics of three- rather than two-dimensional space: the projective images are highly variable, whereas the three-dimensional distal stimuli remain constant. Indeed, the perceptual sistem probably uses the effects of motion to help determine the invariant structure of threedimensional space. The present experiment indicates, however, that such motion is not strictly necessary to obtain a perceptual representation of threedimensional space.

The hypothesis that perceptual processes operate primarily upon three- rather than two-dimensional representations has long been advocated by Gibson (e.g. 1966) and more recently by Attneave (1972; Attneave \& Block, 1973). The same point has also been suggested in a different way by Weisstein and Harris (1974), who demonstrated that lines embedded in a three-dimensional pattern are more difficult to mask than are lines in a two-dimensional pattern. Roberts (1965) has demonstrated how automatic systems for recognizing solid objects can work more efticiently on three- than on two-dimensional representations of the input patterns. The present experiment contributes some empirical support for the hypothesis that the visual system also operates by this approach.

If shape constancy was achieved in this experiment but has failed in many other laboratory studies, the most obvious explanation for the discrepancy is that the stimulus displays used in this experiment provided a richer context of depth cues than has usually been available. The more spatial relations displayed in the projective pattern. the better determined is the transformation that maps the projective plane into the objective three-dimensional space. The finding that there was no main effect produced by rotating the slides $180 \mathrm{deg}$ suggests, in addition, that the perception of shape was based mainly on geometric relationships that are independent of familiarity with specific spatial orientations.

The question of how the visual system is able to determine the appropriate projective transformation with which to interpret the projective image remains a tantalizing problem for future research.

\section{REFERENCE NOTES}

1. Hake, H. W. The constancy legends. Presidential address. Miduestern Psychological Association, Cincinnati, May 1970.

2. Harm, $O$. J. Unpublished doctoral dissertation, Vanderbilt University. In preparation.

\section{REFERENCES}

Attneave, F. Representation of physical space. In A. W. Melton and E. Martin (Eds.), Coding processes in human memory. Washington. D.C: Winston, 1972.

Attneave. F.. \& Block, G. Apparent movement in tridimensional space. Perception \& Psychophysics, 1973, 13, 301-307.

BECK, J.. \& GiBson, J. J. The relation of apparent shape to apparent slant in the perception of objects. Joumal of Experimental Psychology. 1955. 50. 125-133.

Epstein, W., \& Park, J. N. Shape constancy: Functional relationships and theoretical formulations. Psychological Bulletin, 1963, 60, 265-288.

GiBson. J. J. The senses considered as perceptual systems. Boston: Houghton Miftlin. 1966.

HAKE, H. W. Form discriminatior and the invariance of form In L. Uhr (Ed.), Pattern recognition. New York: Wiley, 1\%66. (Originally published as Contributions of psycholugy, to the study of patterm vision. WADC Technical Report 57-621. Wright Air Development Center, 1957.)

Hochelerg, J. Perception: 1I. Shape and movement. In J. W. Kling and L. A. Riggs (Eds.). Woodworth and Schlosberg's Experimental psychology' (3rd ed.). New' York: Holt, Rinehart and Winston, 1972.

Leibowitz, H., \& Bourne, L. E. Time and intensity as determiners of perceived shape. Journal of Experimental Psychology. 1956, 51, 277-281.

RoBERTs, L. G. Machine perception of three-dimensional solids. In J. T. Tippett. D. A. Berkowitz. L. C. Clapp, C. J. Koester, and A. Vanderburgh. Jr. (Eds.). Optical and electro-optical information-processing. Cambridge: M.I.T. Press, 1965.

Thocless. R. H. Phenomenal regression to the real object. Part I. British Joumal of Psychology, 1931, 21. 339-359.

WeIsSTEIN. N.. \& HarRis, C. S. Visual detection of line segments: An object-superiority effect. Science, 1974, 186, 752-755.

\section{NOTES}

1. In retrospect. it would have been more appropriate to use 36 different polygons, one for each combination of objective and projective angles. in order to preclude the possibility that the 
subjects might learn to recognize the polygon associated with a given objective angle. Nevertheless. since the subjects were given no feedback about the correct responses, the greater correspondences of both objective and projective judgments with the actual objective angles could not be due to this characteristic of the stimuli. As a check on whether the difference between the objective and projective judgments was magnified by the reduced physical variability associated with the objective angles, we examined the sequences of trials within a given condition and also the four separate blocks of trials for each subject. but were able to detect no indication of an increasing correspondence of the judgments with the objective angles. The overall similarity in the magnitude of the correspondence with the objective angles under both objective and projective judgments provides an additional indication that there was no reliable effect associated with this characteristic of the stimuli.

2. The $1 / 2$-sec exposure duration was selected somewhat arbitrarily to provide a view that was sufficiently long for the stimulus to be easily visible but that was too brief for the subject to rationally analyze the stimulus while it was physically present. A basic question. however. concerns the dependence of the results on the exposure duration. Leibowitz and Bourne (1956) found that as the exposure duration was reduced below $1 / 2$ sec the tendency toward shape constancy was also reduced. Later experiments in our laboratory (Harm, Note 2), though, have demonstrated that reaction times for discriminating three-dimensional shapes are faster than those for discriminating two-dimensional shapes-thus suggesting that information about three-dimensional space is obtained earlier in the perceptual process and that discriminations of two-dimensional projective shapes require additional perceptual analysis.

(Received for publication October 21, 1974; revision received January 8,1975 .) 